\title{
Optics and Solid State at Laboratories RCA Ltd., Zurich
}

Laboratories RCA Ltd., the European technical centre of RCA Corporation, with a total manpower of about 75 , has about 20 scientists and 25 supporting technical staff engaged on fundamental studies in optics and solid state physics - in the development of new concepts in the first of these fields and new processes in the second. In addition, close cooperation with European licencees is maintained, particularly in the area of colour TV. One project only in each of the two fields mentioned will be treated in some detail, namely "Light scattering - a tool in research and application" and "Diffraction optics embossed plastic as microfiche". Other projects can be summarized under the four headings set out below.

Microlithography. The interest here is in preparing and controlling line patterns in the micron range in various materials. One approach is sputter deposition and sputter etching by plasma and reactive gases. Etch selectivity for $\mathrm{Si}, \mathrm{SiO}_{2}$ and various metals, and also profile control are of prime importance. A second approach is the preparation and projection of micron dimension patterns by deep UV photolithography and by accurate computer controlled $x-y$ motion. These patterns are being developed for silicon integrated circuit technology and for special optical components like filters etc. (see below).

Optical Methods for Testing. A number of optical methods have been developed, based on scattering, diffraction and interference of light, which allow automatic in-process control of critical parameters in silicon IC manufacturing. Control of line widths during etching, control of film thickness during deposition, control of defects on wafers and masks and control of wafer flatness are typical applications (see also below).

Polymers. The best way of understanding the conduction mechanism in polymers is probably by studying the polydiacetylenes, a class of polymers which can be grown in single crystalline form. Electrical measurements are combined with detailed optical studies. Polyacetylenes are also being studied in detail using con- ventional techniques, with the main emphasis on doping with halogens and oxygen, a process, which influences conduction by many orders of magnitude. In addition, attempts have been made to develop well conducting polymers by molecular doping which yields both $\mathrm{n}$ - and $\mathrm{p}$-type material.

Studies of Low Dimensional Materials. One- and two-dimensional solids are being investigated by optical and ultrasonic methods. In particular, materials which undergo phase transitions (structural, ferroelectric, magnetic) and chain- or layer-structure materials undergoing a Peierls transition have been investigated both experimentally and theoretically. The aim is to understand the interaction mechanisms involved, the electronic band structure and the phonon and magnon spectra for both bulk and surface modes.

\section{Light Scattering - a Tool in Research and Application}

Light scattering using advanced laser technology is one of the most suitable techniques for studying vibrational spectra as well as magnetic and electronic excitations in solids. The basic principle of light scattering can best be illustrated for the case of a crystalline solid with two atoms per unit cell as, e.g. silicon. The vibrational excitations of such a crystal consist of acoustic phonons (lattice vibrations) which in the long-wavelength limit are equivalent to sound waves, and optical lattice vibrations in which the atoms within a unit cell move against each other. Coupling of electromagnetic radiation to these lattice vibrations is only possible for long wavelengths equivalent to small momenta, when the momentum of the incoming photon is small compared to the maximum phonon momentum. The coupling mechanism can result in the direct absorption of light by phonons or in the scattering of light by phonons. In the scattering process, the incoming photon can be scattered inelastically such that the scattered photon is of different energy or quasielastically with a very small change of energy. The momentum and thus the energy of the measured excitation depends in either case on the scattering geometry.

\section{W. J. Merz, Zurich}

There are two experimental techniques: Raman scattering measures the inelastic scattering of light from optical lattice vibrations or other excitations of comparable energy. Brillouin scattering measures the inelastic scattering from acoustic lattice vibrations or other excitations of comparable energy as well as quasi-elastic scattering from entropy fluctuations. Experimental observations of scattered light requires high spectral resolution and extremely high contrast. In Raman scattering, this is achieved by a triple spectrometer (three gratings in series). In Brillouin scattering new concepts developed at RCA Zurich include the multipass technique in which the beam of scattered light is passed up to five times through a Fabry-Perot interferometer thereby increasing the contrast by about six orders of magnitude. More recently, a tandem arrangement of two multipass interferometers of different free spectral range has been developed which yields a much larger total free spectral range.

As a specific exemple of the outcome of this research, it has been found that $\mathrm{SrTiO}_{3}$ undergoes a second order structural transition at about $108^{\circ} \mathrm{K}$ which manifests itself in a softening of an optical lattice mode (frequency goes to zero at $T_{c}$ ). Raman measurements have shown that the temperature dependence of the soft mode frequency does not follow the usual Landau law but exactly parallels the behaviour of the static order parameter as measured by electron spin resonance. These results provide a valuable link between dynamic and static critical phenomena.

More recently we have investigated electrically driven structural phase transitions. Peierls transitions, in nearly one- or two-dimensional systems. The occurrence of a periodic lattice distortion along the chain or within the plane is triggered by a dimensionally enhanced dielectric function; i.e., in the distorted state the coupled electron-phonon system carries specific collective excitations such as the Raman active charge density amplitude mode. The linear chain salt $\mathrm{K}_{2} \mathrm{Pt}(\mathrm{CN})_{4} \mathrm{Br}_{0.3} \cdot 3.2 \mathrm{H}_{2} \mathrm{O}$ was found to have a temperature inde- 

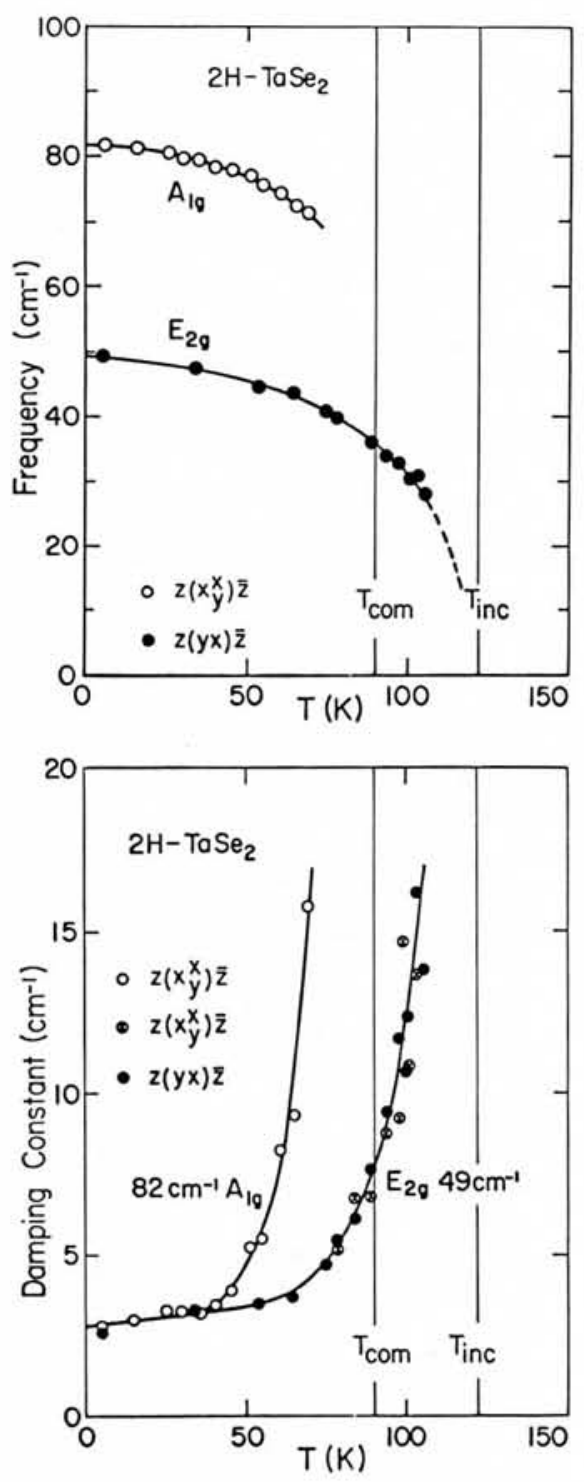

Fig. 1 - Frequency (upper part) and damping constant (lower part) of charge density modes in $2 \mathrm{H}-\mathrm{TaSe}{ }_{2}$ vs. temperature.

pendent amplitude mode which indicates that the Peierls transition is present at all temperatures. The twodimensional layer compound $2 \mathrm{H}-\mathrm{TaSe}_{2}$ was found to show at $120^{\circ} \mathrm{K}$ a distorted state which is incommensurate with the original lattice and at $90^{\circ} \mathrm{K}$ a state with a commensurate superstructure. As can be seen in Fig. 1; there are two modes of $A_{7}$ and $E_{g}$ symmetry which soften in frequency and increase in width due to additional damping.

In addition to the ability to obtain Brillouin spectra from highly absorbing materials and small transparent crystals, we are now able to study a number of other excitations thanks to the newly developed Fa'oryPerot interferometer. For example, acoustic magnons or spin waves propagating in the volume of magneti- cally ordered crystals can be studied yielding a wealth of information on magnetic properties (exchange constant, anisotropy fields, magnetophonon coupling, dispersive and dissipative effects).

A very exciting discovery is the fact that many materials have been found which show a previously undetected mode of quasi-elastically scattered light (i.e. near zero frequency) which is in no way related to a phase transition. For example, GaP shows at room temperature a double structure of a narrow component ( $\sim 10 \mathrm{GHz}$ ) and a broad component ( $\sim 50 \mathrm{GHz}$ ). Silicon, on the other hand, shows one broad component. The measured values seem to agree quantitatively with theoretical predictions for scattering from entropy fluctuations. Brillouin scattering was also observed from thermally excited surface phonons in the $10 \mathrm{GHz}$ region in opaque materials. Also surface sound velocities could be directly evaluated for a number of semiconductors, semimetals and crystalline and amorphous metals. The composite Brillouin spectrum of silicon (Fig. 2) shows a central peak due to scattering from entropy fluctuations extending out to about $50 \mathrm{GHz}$, the surface phonon line $R$, and the bulk phonons $T$ (transversal) and $\mathrm{L}$ (longitudinal).

In addition to its use in fundamental research, light scattering can also find many practical applications. Two examples from the field of silicon device processing illustrate this. Contactless and small area determination of carrier concentrations can be accomplished by light scattering from free carriers. This works particularly well for p-type silicon due to scattering within the sub-bands of the valence band. Since the penetration

Fig. 2 - Brillouin spectrum of silicon. of visible laser light is of the order of one micron, the method is well suited to the characterization of thin diffused or ion-implanted layers. The second example employs the effect of elastic light scattering from imperfections or defects located on a silicon wafer surface or just beneath it. A laser scanner has been developed which displays on a storage screen, scattered light from a wafer or mask due to scattering from defects (dust, scratches, dislocations etc.). Also pinholes in chrome masks can be tested. This tool has been found very useful for the fast testing of wafers in a routine manner.

\section{Diffraction Optics - \\ Embossed Plastic as Microfiche}

The use of surface-relief structures for the recording and replication of information has spanned many thousands of years. Early examples such as ancient seals can be found in many museums ; modern examples such as gramophone records are found in most homes. For the past several years, we have been investigating methods for recording and replicating microimages using surfacerelief phase diffraction grating structures. This work has led to the development of a new medium for micropublishing, in which black and white and colour images are produced by hot-embossing a metal master into transparent thermoplastic sheet. The images can still be read out in conventional slide projectors and microfilm readers. Whereas normal photographic emulsions produce images by selectively absorbing the unwanted light in silver or dye layers, this new technique uses optimised diffraction gratings to diffract the unwanted light out of the projection lens

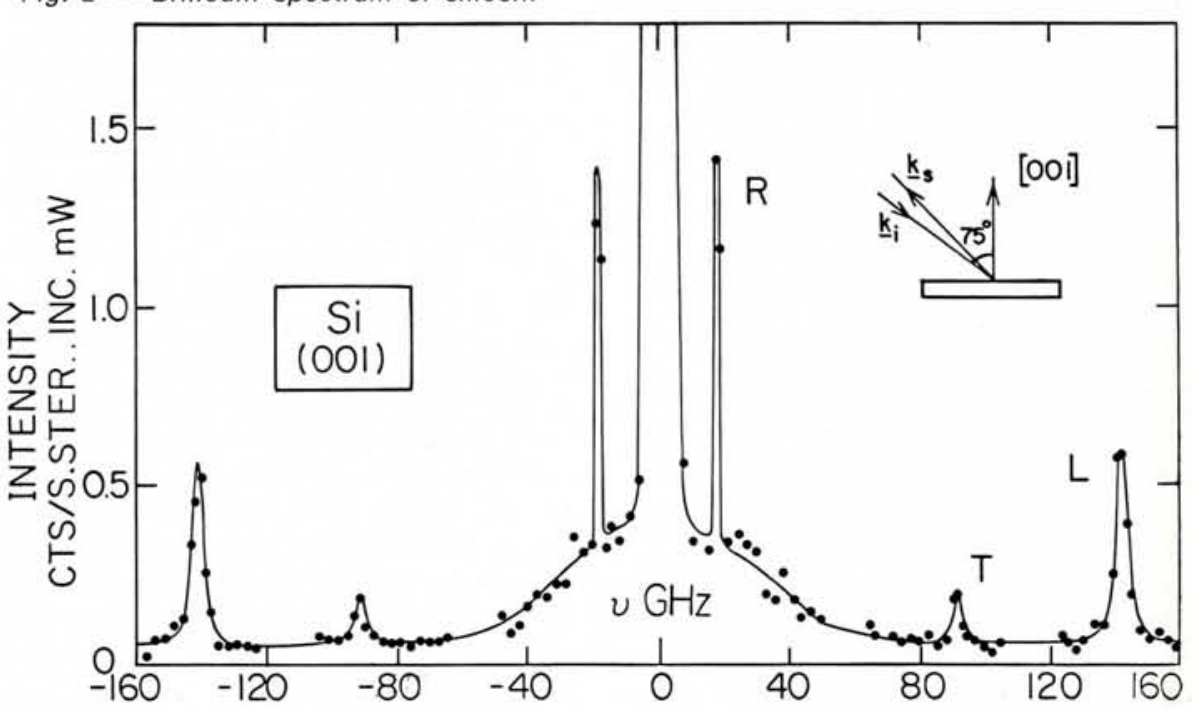



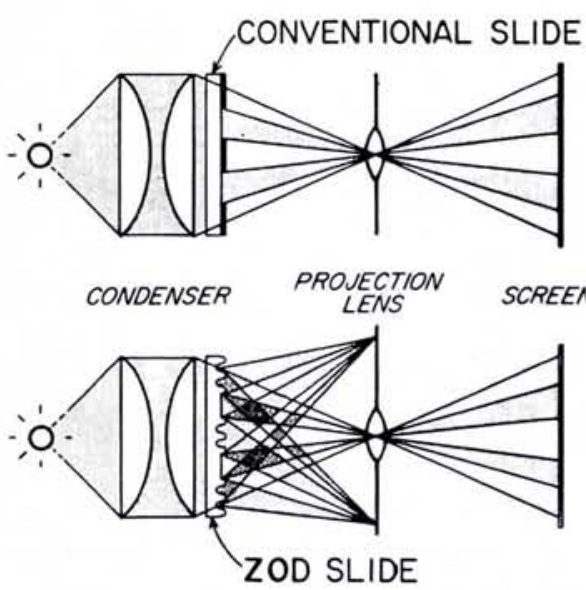

Fig. 3 - Read-out of conventional and $Z O D$ surface relief images in a standard projector.

aperture (see Fig. 3). Provided the grating period is sufficiently fine (typically about $1.5 \mu \mathrm{m}$ ) only the zero-order, undiffracted light passes through the lens to form an image on the screen these images have thus been named ZOD images (from Zero Order Diffraction).

Under white light illumination, the intensity and spectral composition of the light transmitted in the zero-order is determined by the profile and amplitude of the relief grating structure. Black and white images are reproduced using a crossed sinewave relief. For colour, squarewave gratings of different depths generate the yellow, magenta and cyan primaries for a subtractive colour scheme. Fig. 4 shows SEM enlargements of one of these three embossed plastic layers, which are superimposed to form such a tripack ZOD colour image. In fabrication, durable metal masters of the required relief structures are produced by photolithographic and electroplating techniques, and are then used for hot embossing into clear plastic sheet such as PVC.

The main application of ZOD image technology is in micropublishing, where information (journals, catalogues, price lists, etc.) is distributed in microfiche format. The system offers the valuable combination of full compatibility with existing display equipment, together with a low cost per copy for colour and black and white embossed replicas in volume. Resolution is similar to that of conventional micrographic recording materials and colour quality is comparable with that of colour microfilm emulsions. Since they are relief structures, ZOD images do not bleach or fade and are thus ideal for the archival storage of colour images - an application for which alternative recording media are currently not sufficiently stable. Diffractive structures such as these may also find applications as colour filters and beam splitters in areas where low cost and low weight are important considerations.

ZOD surface relief structures have also been used as the basis of a novel thermal recording scheme. The recording medium is plastic sheet pre-embossed with an optimized sinewave relief profile which would reconstruct black in read-out. A scanning, focused laser beam is used to write or contact-print images by selectively heating the plastic surface, resulting in plastic flow and a local levelling of the grating relief. Sensitization to the recording wavelength is achieved by incorporating dye into the plastic as a thin surface layer, enabling sensitization to any wavelength region in the visible to be readily achieved. Written areas are then coloured by the dye ; unwritten areas remain black. Applications include updateable microfiche and slides, and instant image recording.

A theoretical understanding of the diffraction properties of transmission phase gratings with grating constants and rectangular groove depths of the order of the light wavelength has been developed using rigorous diffraction theory. The scalar treatment based upon Huygen's and Kirchhoff approximations breaks down for such fine, deep gratings and the problem must be treated by numerical solution of Maxwell's equations. The theoretical predictions then agree closely with the measured diffraction efficiencies and can be used to optimize the rectangular groove grating parameters for a given filter application. Additionally, the properties and fabrication of multi-

\section{Scholarships at the University of Nijmegen}

The Faculty of Science of the University of Nijmegen is offering research fellowships to graduate students who hold a master's degree in science or its equivalent and who have at least one year's experience in research. Valid for 12 months they will initially be applicable to the academic year 1980-81.

Fellows are expected to carry out full-time research in one of the University's laboratories. A working knowledge of the Dutch language is not required but proficiency in English, French or German is necessary. The net monthly allowance will be h.FI. 1750.

There are openings in the: Dept. of Informatics and Mathematics Section; in the experimental Depts. of Atomic
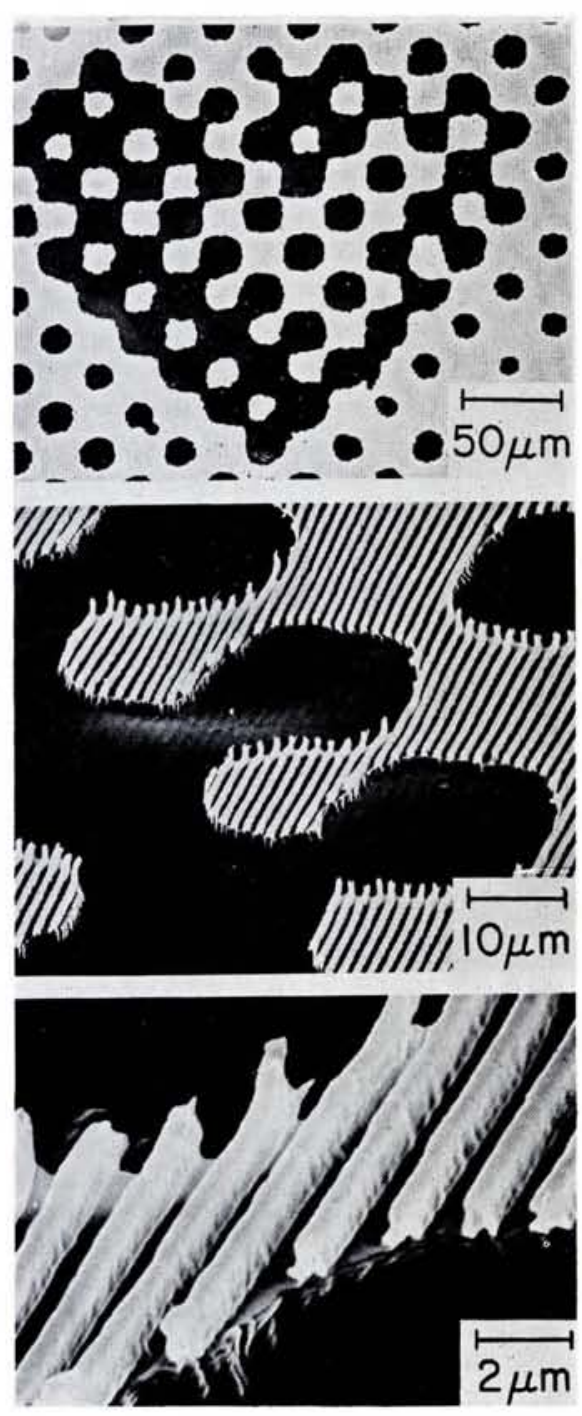

Fig. 4 - SEM enlargements of a colour ZOD image embossed in PVC.

level profiles are being investigated for applications in areas other than micro-imaging.

and Molecular Physics, High Energy Physics, Medical Physics and Biophysics, Solid State Physics and Solid State Chemistry; in the theoretical Depts. of Mechanics, High Energy Physics, Solid State Physics, and Astronomy; and in the Chemistry Section in the Depts. of Molecular Spectroscopy and Physical Chemistry.

Information concerning the research objectives and further details on the arrangements can be obtained direct from the Secretary of the Fellowship Committee, Faculty of Science, Toernooiveld, Nijmegen, The Netherlands. the EPS Secretariat in Geneva. Note that applications need to be made as soon as possible preferably even before 1 December, 1979. Application forms are available from 